\title{
Correction to: A DHX9-IncRNA-MDM2 interaction regulates cell invasion and angiogenesis of cervical cancer
}

\author{
Xiangya Ding $\mathbb{1}^{1,2,3} \cdot$ Xuemei Jia ${ }^{4} \cdot$ Cong Wang ${ }^{5} \cdot$ Jingyun $\mathrm{Xu}^{3} \cdot$ Shou-Jiang Gao ${ }^{3,6,7} \cdot$ Chun $\mathrm{Lu}^{1,2,3}$
}

Published online: 25 February 2019

(c) ADMC Associazione Differenziamento e Morte Cellulare 2019

\section{Correction to: Cell Death \& Differentiation (2018) 25}

https://doi.org/10.1038/s41418-018-0242-0

published online 05th December 2018

Since publication of the article the authors found two small issues with Fig. 2c ( $\mathrm{SiHa} / 24 \mathrm{~h} /$ Untreated) and Fig. $\mathrm{S} 7 \mathrm{a}(\mathrm{HeLa} / 12 \mathrm{~h} / \mathrm{sh} 1 \mathrm{DHX} 9)$. The correct Figures are given below, and do not change the conclusions.

The authors apologize for these mistakes.

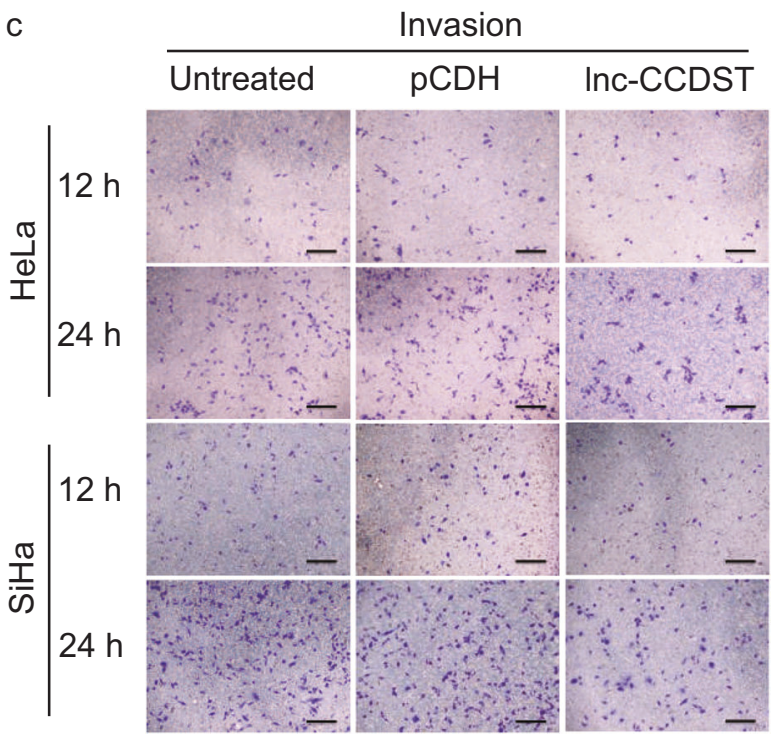

Fig 2
Edited by M. Piacentini

The original article can be found online at https://doi.org/10.1038/ s41418-018-0242-0.

Chun Lu

clu@njmu.edu.cn

1 State Key Laboratory of Reproductive Medicine, Nanjing Medical University, Nanjing, People's Republic of China

2 Key Laboratory of Pathogen Biology of Jiangsu Province, Nanjing Medical University, Nanjing, People's Republic of China

3 Department of Microbiology, Nanjing Medical University, Nanjing, People's Republic of China

4 Department of Gynecology, Nanjing Maternity and Child Health Hospital, the Affiliated Obstetrics and Gynaecology Hospital of Nanjing Medical University, Nanjing, People's Republic of China

5 Department of Pathology, the First Affiliated Hospital of Nanjing Medical University, Nanjing, People's Republic of China

6 Laboratory of Human Virology and Oncology, Shantou University Medical College, Shantou, Guangdong, China

7 UPMC Hillman Cancer Center, Department of Microbiology and Molecular Genetics, University of Pittsburgh, Pittsburgh, PA 15232, USA 